\title{
Low-temperature magnetism in YbBiPt
}

\author{
A. Amato \\ Institut für Mittelenergiephysik der Eidgenössische Technische Hochschule Zürich, \\ CH-5232 Villigen PSI, Switzerland \\ P. C. Canfield \\ Los Alamos National Laboratory, Los Alamos, New Mexico 87545 \\ R. Feyerherm \\ Institut für Mittelenergiephysik der Eidgenössische Technische Hochschule Zürich, \\ CH-5232 Villigen PSI, Switzerland \\ Z. Fisk \\ Los Alamos National Laboratory, Los Alamos, New Mexico 87545 \\ F. N. Gygax \\ Institut für Mittelenergiephysik der Eidgenössische Technisch Hochschule Zürich, \\ CH-5232 Villigen PSI, Switzerland \\ R. H. Heffner \\ Los Alamos National Laboratory, Los Alamos, New Mexico 87545 \\ D. E. MacLaughlin* \\ Kamerlingh Onnes Laboratorium, University of Leiden, 2300 RA Leiden, The Netherlands
}

H. R. Ott

Laboratorium für Festkörperphysik, Eidgenössische Technische Hochschule Hönggerberg, CH-8093 Zürich, Switzerland

A. Schenck

Institut für Mittelenergiephysik der Eidgenössische Technische Hochschule Zürich, CH-5232 Villigen PSI, Switzerland

J. D. Thompson

Los Alamos National Laboratory, Los Alamos, New Mexico 87545

(Received 13 April 1992)

\begin{abstract}
Positive-muon $\left(\mu^{+}\right)$spin-relaxation experiments have been carried out in a pressed-powder sample of the low-carrier-density heavy-electron system YbBiPt. Spatially inhomogeneous and disordered static $\mathrm{Yb}$ magnetism is observed below $\sim 0.5 \mathrm{~K}$, with a strongly reduced $\mathrm{Yb}$ moment of $\sim 0.1 \mu_{B}$ over $\sim 50 \%$ of the sample volume at $T=0.06 \mathrm{~K}$. Substantial $\mu^{+}$spin-lattice relaxation, rarely observed in heavy-electron systems, suggests anomalously slow $\mathrm{Yb}$ spin fluctuations. Our data are reminiscent of $\mu^{+}$behavior in spin glasses, and raise the question of whether the large low-temperature specific heat in $\mathrm{YbBiPt}$ is due in part to low-lying magnetic excitations.
\end{abstract}

The $R \mathrm{BiPt}$ ( $R=$ rare earth) series of ternary intermetallic compounds exhibits a rather unique evolution from insulating to weak metallic behavior with increasing rareearth atomic number. ${ }^{1}$ Among these materials YbBiPt is of particular interest. Measurements of the specific heat $c_{p}$ is flux-grown single crystals of this compound ${ }^{2}$ yield an enormous Sommerfeld coefficient $\gamma=c_{p} / T \simeq 8 \mathrm{~J} \mathrm{~mol}^{-1}$ $\mathrm{K}^{-2}$ below $\sim 0.4 \mathrm{~K}$, suggestive of an extremely massive itinerant-electron state. A low characteristic temperature is indicated by an entropy release of $R \ln 2$ between zero and $1 \mathrm{~K}$. A cusp in the ac susceptibility at $0.4 \mathrm{~K}$ indicates some sort of low-temperature magnetic phase which seems to coexist with the heavy-electron state. The specific heat of crushed powders of YbBiPt (Ref. 3) is nearly the same as in unstrained material above $0.5 \mathrm{~K}$, but below this temperature the $c_{p} / T$ ratio is reduced by approximately $50 \%$ and there is no clear evidence for a magnetic phase transition.

These results indicate that $\mathrm{YbBiPt}$ is situated on the boundary between magnetic and heavy-electron behavior with respect to the $\mathrm{Yb}$ spin degrees of freedom. Questions are raised concerning (1) the stability of the $\mathrm{Yb}$ moment, (2) the nature of a heavy-electron state which can evolve from a low-carrier-density metal at high temperatures, and (3) the competition between Kondo singlet formation and Rudermann-Kittel-Kasuya-Yosida interactions.

This paper presents the results of positive-muon spin rotation $\left(\mu^{+} \mathrm{SR}\right)$ experiments which bear on the above 
questions. Like other magnetic resonance techniques, $\mu^{+}$SR provides a microscopic probe of local static and dynamic magnetic behavior in solids. ${ }^{4}$ In the timedifferential $\mu^{+}$SR method used in these studies spinpolarized positive muons $\left(\mu^{+}\right)$are implanted into the sample, and the subsequent precession and relaxation of the $\mu^{+}$polarization is monitored. In high transverse applied field $H_{\perp}$ (applied field $\mathbf{H}_{0}$ perpendicular to the $\mu^{+}$ polarization $\mathbf{p}_{\mu}$ ) the relaxation rate is usually determined by the inhomogeneous distribution of static local fields at $\mu^{+}$sites. In high longitudinal field $H_{\|}\left(\mathbf{H}_{0} \| \mathrm{p}_{\mu}\right)$ the relaxation is due to thermal fluctuations of the local fields (spin-lattice relaxation), 4.5 and in zero applied field both dynamic and static relaxation components can be observed. ${ }^{5.6}$ Zero to high field crossover occurs when $\left|\mathbf{H}_{0}\right|$ is of the order of the static local field distribution width $\Delta H_{L}$. One complication associated with the technique is the fact that the number and location of muon stopping sites may not be known.

Polycrystals of $\mathrm{YbBiPt}$ were prepared by the fluxgrowth technique. ${ }^{7}$ They were crushed, mixed with a small amount of GE 7031 varnish, and pressed to form a pellet with $\sim 80 \%$ of the theoretical density. The $\mu^{+} \mathrm{SR}$ experiments were carried out at the Low Temperature Facility of the Paul Scherrer Institute, Villigen, Switzerland, over the temperature range $0.06-300 \mathrm{~K}$.

Figure 1 shows the observed time-differential relaxation function $G_{Z F}(t)$ in zero applied field for $T=0.06 \mathrm{~K}$. There is no sign of a well-defined precession frequency or frequencies, indicating that static YB magnetism, if present, is either spatially disordered or incommensurate. The two-component structure evident in Fig. 1 is observed for $T \lesssim 20 \mathrm{~K}$. Good fits were obtained to a sum of fast and slow components of the form $G_{\mathrm{ZF}}(t)=A_{f} g_{f}(t)$ $+A_{s} \exp \left(-\lambda_{s} t\right)$, where $A_{f}$ and $A_{s}$ are the amplitudes of

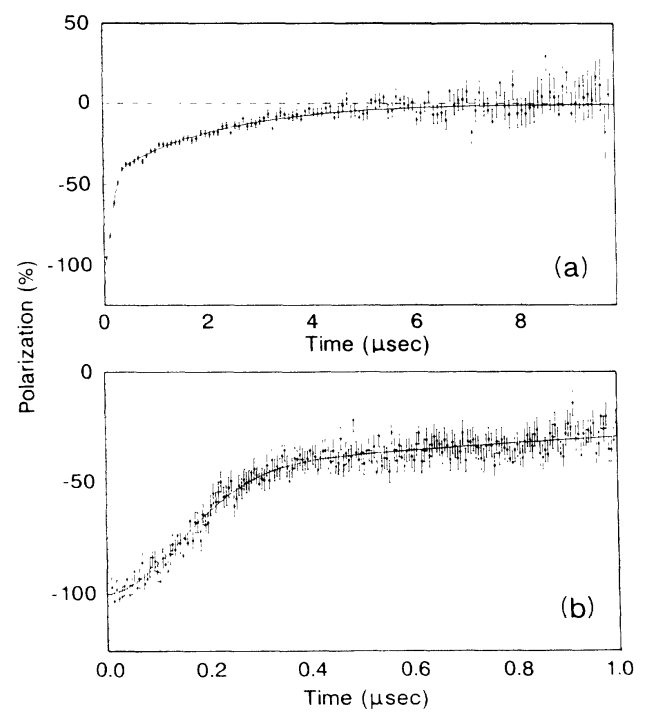

FIG. 1. Zero-field time-differential $\mu^{+} \mathrm{SR}$ histogram in YbBiPt, $T=0.060 \mathrm{~K}$. (a) Time range $0-8 \mu \mathrm{s}$. The two-component structure is easily recognizable. (b) Time range $0-10 \mu \mathrm{s}$, showing early part of the relaxation function. The Gaussian nature of the fast component is evident. the fast and slow components, respectively, and $\lambda_{s}$ is the exponential rate of the slow component. The form $g_{f}(t)$ of the fast component was observed to change abruptly from Gaussian $\left[g_{f}=\exp \left(-\sigma_{f}^{2} t^{2}\right)\right]$ below $0.5 \mathrm{~K}$ (cf. Fig. 1) to exponential $\left[g_{f}=\exp \left(-\lambda_{f} t\right)\right]$ above this temperature. The relaxation rates and the component amplitudes $A_{f}$ and $A_{s}$ were varied for best fit, with the total amplitude $A_{f}+A_{s}$ held at a value obtained from weak transverse-field calibration runs.

The temperature dependence of $A_{f}$ and $A_{s}$ is given in Fig. 2. With increasing temperature $A_{f}$ decreases monotonically (with a corresponding increase of $A_{s}$ ). By $\sim 0.5$ $\mathrm{K}$ half the weight of the fast component has been transferred to the slow component. This is near the temperature at which the Gaussian to exponential crossover occurs, and where $c_{p} / T$ of the crushed sample shows a maximum. ${ }^{3}$ Above $30 \mathrm{~K} A_{f} \simeq 0$.

The temperature dependence of the fast-component Gaussian relaxation rate $\sigma_{f}$ below $0.5 \mathrm{~K}$ is given in Fig. 3 (a). Measurement of the fast-component exponential relaxation rate $\lambda_{f}$ above $0.5 \mathrm{~K}$ was only possible below $\sim 0.7$ $\mathrm{K}$, due to the small values of $A_{f}$ for higher temperatures (Fig. 2); in this limited temperature range $\lambda_{f} \simeq 3 \mu \mathrm{s}^{-1}$. The slow-component exponential relaxation rate $\lambda_{s}$ is given in Fig. 3(b). Assuming that the relaxation is in the motionally narrowed limit, the observed decrease in $\lambda_{s}$ with increasing temperature up to $\sim 1 \mathrm{~K}$ [Fig. 3(b)] indicates that the $\mu^{+}$local-field fluctuation rate increases with temperature over this range. The rapid decrease of $\lambda_{s}$ above $100 \mathrm{~K}$ probably signals the onset of $\mu^{+}$diffusion.

Measurements in a longitudinal field $H_{\|}=5 \mathrm{kOe}$ between 0.1 and $1.06 \mathrm{~K}$ revealed substantial $\mu^{+}$spin-lattice relaxation. An example for $T=0.06 \mathrm{~K}$ is given in Fig. 4 . Observable spin-lattice relaxation is rare in heavyelectron and related systems, and indicates low-frequency fluctuations of an appreciable $\mathrm{Yb}$ moment. The exponential form $G_{\|}(t)=A_{\|} \exp \left(-\lambda_{\|} t\right)$ gave good fits to the data. The amplitude $A_{\|}$was reduced to $\sim 0.4\left(A_{f}+A_{s}\right)_{\mathrm{ZF}}$ (cf. Fig. 4), independent of temperature, and the relaxation rate $\lambda_{\|}$varied only slightly, from $0.75(5) \mu \mathrm{s}^{-1}$ at $0.1 \mathrm{~K}$ to $1.0(1) \mu \mathrm{s}^{-1}$ at $1.06 \mathrm{~K}$.

Interpretation of these data depends on knowledge of the number of $\mu^{+}$stopping sites, so we begin by consider-

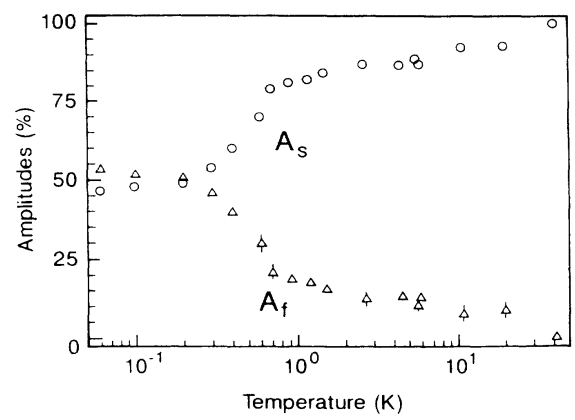

FIG. 2. Temperature dependence of zero-field $\mu^{+} \mathrm{SR}$ fastand slow-component amplitudes in YbBiPt. $\triangle$ : fast-component amplitude $A_{f}$. O: slow-component amplitude $A_{s}$. Relative values of $A_{f}$ and $A_{s}$ are measures of the volume fractions of "frozen-spin" and "paramagnetic" domains, respectively. 

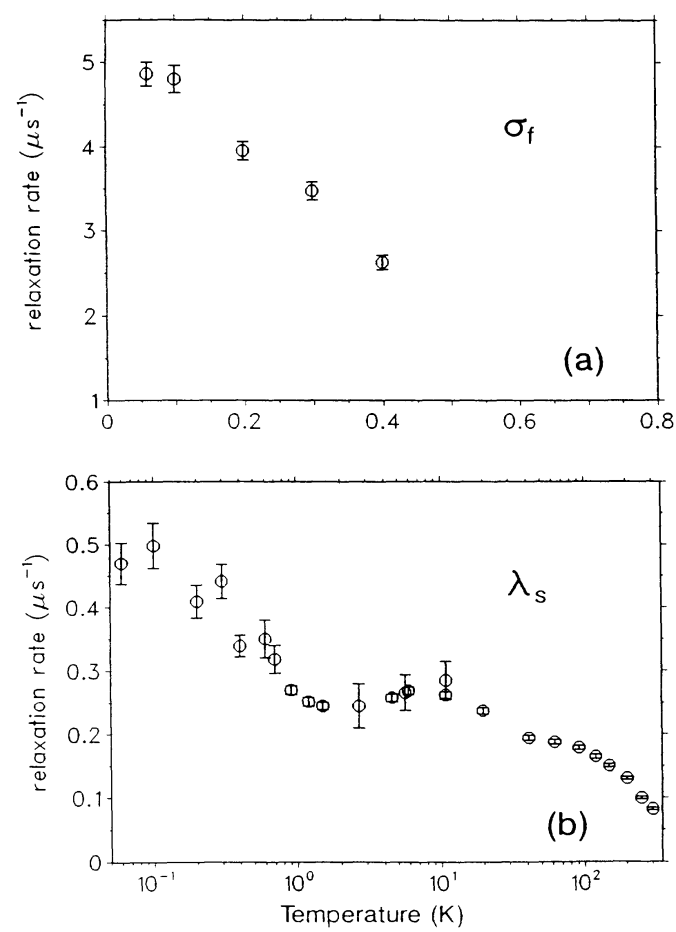

FIG. 3. Temperature dependence of zero-field fast- and slow-component $\mu^{+} \mathrm{SR}$ relaxation rates in YbBiPt. (a) Fastcomponent Gaussian relaxation rate $\sigma_{f}=\sigma_{\mathrm{KT}}$ below $0.5 \mathrm{~K}$. (b) Slow-component exponential relaxation rate $\lambda_{s}$. The fastcomponent exponential relaxation rate $\lambda_{f}=3 \mu \mathrm{s}^{-1}$ between 0.5 and $0.7 \mathrm{~K}$ is not shown.

ing this question. $\mu^{+} \mathrm{SR}$ was carried out in a transverse field $H_{\perp}=2.9 \mathrm{kOe}$ at temperatures above $2.5 \mathrm{~K}$. These measurements, which will be reported elsewhere in more detail, yielded a single signal with asymmetric broadening typical of an anisotropic Knight shift powder pattern. There is therefore only one $\mu^{+}$site, and this site has lower than tetrahedral symmetry. Comparing these data with simulated $\mu^{+}$powder spectra, we tentatively identify the muon site as an off-center position shifted $0.4 \AA$ from the $\left(\frac{1}{2}, \frac{1}{2}, \frac{1}{2}\right)$ point toward a nearest-neighbor $\mathrm{Bi}$ in the fcc MgAgAs (half-Heusler) structure.

The existence of two $\mu^{+}$SR signal components in zero field then suggests the existence of two different magnetic

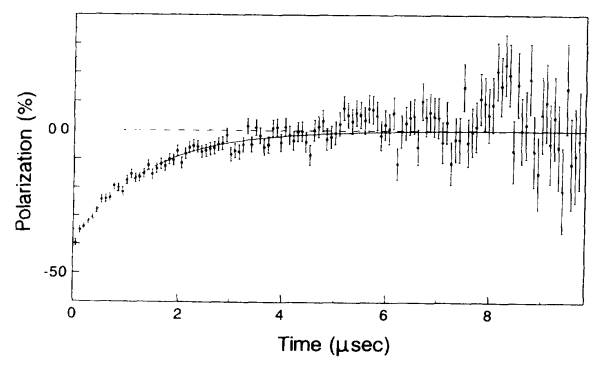

FIG. 4. Time-differential $\mu^{+} \mathrm{SR}$ histogram in YbBiPt, longitudinal field $H_{\|}=5 \mathrm{kOe}$, temperature $0.06 \mathrm{~K}$. Note the reduced amplitude compared to the zero-field histograms of Fig. 1. environments or domains. The size of these domains is unknown, except that they must be somewhat larger than a few unit cell volumes. The fast Gaussian component below $0.5 \mathrm{~K}$ must originate from "frozen-spin" domains characterized by a static or quasistatic field component distribution with zero mean and rms width $\Delta H_{L}=\sigma_{f} / \gamma_{\mu}$, where $\gamma_{\mu}$ is the $\mu^{+}$gyromagnetic ratio. This result rules out a nonmagnetic ground state for $\sim 50 \%$ of our sample. A Gaussian distribution is expected for a regular lattice of randomly oriented moments, ${ }^{6,8}$ but not for an incommensurate periodic spin arrangement.

For random moment orientations the zero-field $\mu^{+} \mathrm{SR}$ signal should be given by the static Kubo-Toyabe (KT) function $^{6} \quad G_{\mathrm{KT}}(t)=\frac{1}{3}+\frac{2}{3}\left(1-\sigma_{\mathrm{KT}}^{2} t^{2}\right) \exp \left(-\frac{1}{2} \sigma_{\mathrm{KT}}^{2} t^{2}\right)$. The short-time behavior of $G_{\mathrm{KT}}(t)$ is Gaussian, in agreement with our data, with $\sigma_{f}=\sigma_{K T}$. We do not observe the typical KT recovery to $\frac{1}{3}$ at long times, which indicates that the recovery signal is damped by spin-lattice relaxation.

The static disordered magnetism in frozen-spin domains indicated by the Gaussian form of the rapid component is not likely to be due to lattice defects, given the sharp $x$-ray Bragg lines in our YbBiPt samples. The observed value of $\sigma_{f}$ at low temperatures corresponds to $\Delta H_{L} \simeq 60 \mathrm{Oe}$. Assuming dipolar coupling to a muon at the site described above, a standard Van Vleck second-moment calculation yields $\sim 0.1 \mu_{B}$ for the static $\mathrm{Yb}$ moment. This is consistent with the absence of a nuclear Schottky term in the specific heat, ${ }^{2}$ which would not be observed above $\sim 50$ $\mathrm{mK}$ for this moment value.

The abrupt crossover at $0.5 \mathrm{~K}$ of the fast component from a Gaussian to an exponential form signals the loss of a static spin component and onset of strong thermal fluctuations in the $\mu^{+}$local field in the corresponding domains. (We will continue to use the label "frozen-spin" for these domains even though there is no static spin component above $0.5 \mathrm{~K}$.) In the motionally narrowed limit ${ }^{5,6}$ $\lambda_{f}=2 \sigma_{\mathrm{KT}}^{2} \tau_{c}$, where $\tau_{c}$ is the correlation time of the spin fluctuations. With $\sigma_{\mathrm{KT}}=5 \mu \mathrm{s}^{-1}$ from the low-temperature data, we obtain $\tau_{c} \simeq 2 \times 10^{-7}$ s between 0.5 and 0.7 K.

The slow zero-field component is exponential over the full temperature range $0.06-300 \mathrm{~K}$. This indicates the $\mathrm{ab}$ sence of static $\mathrm{Yb}$ magnetism and motionally narrowed relaxation by spin fluctuations in the corresponding "paramagnetic" domains, for which we obtain $t_{c} \simeq 10^{-8} \mathrm{~s}$ at low temperatures. For a system obeying ordinary spin dynamics with a characteristic energy scale $k_{B} T_{0}$, an order-of-magnitude estimate of $\tau_{c}$ is $\left(k_{B} T_{0} / \hbar\right)^{-1}$ $\sim 10^{-11} \mathrm{~s}$ if we take $T_{0} \sim 0.5 \mathrm{~K}$. The observed correlation times are very much longer than this in both paramagnetic and frozen-spin domains.

The relaxation in a longitudinal field of $5 \mathrm{kOe}$ also indicates the presence of two components, viz., the observed signal and an unobserved fraction. The latter is probably a nonrelaxing constant background due to frozen-spin domains. ${ }^{9}$ Unlike the behavior in zero field, however, the volume fraction of frozen-spin domains in $5 \mathrm{kOe}$ appears to be temperature independent between 0.1 and $1 \mathrm{~K}$. In addition $\lambda_{\|}$is larger than $\lambda_{s}$ in zero field, which can be understood only if $\tau_{c}$ increases with increasing field. Thus 
the presence of an external field $H \sim k_{B} T_{0} / \mu_{B}$, where $T_{0}=0.5 \mathrm{~K}$ as estimated above, seems to alter the microscopic behavior of the sample considerably. The magnetoresistance of $\mathrm{YbBiPt}$ is also found to be very sensitive to small ( $\lesssim 5 \mathrm{kOe}$ ) magnetic fields. ${ }^{2}$

Several conclusions can be drawn from these data.

(1) The observed two-component form of the $\mu^{+}$relaxation function suggests an inhomogeneous distribution of Yb magnetism over two kinds of domains. Similar behavior has been reported in the heavy-electron compounds $\mathrm{CeAl}_{3}$ (Ref. 10) and $\mathrm{URu}_{2} \mathrm{Si}_{2}$ (Ref. 11), where it is regarded as an intrinsic property.

(2) Disordered static magnetism, with a $\mathrm{Yb}$ moment much less than the free-ion value, sets in abruptly below $0.5 \mathrm{~K}$ over about half of the sample. This temperature corresponds to the maximum in the specific heat observed in other crushed-power samples. ${ }^{3}$ The sharp transition precludes the attribution of the more continuous temperature dependence of the frozen-spin volume fraction (Fig. 2) to a distribution of transition temperatures. Taken as a whole the data suggest a percolative process, which attains the percolation threshold at $\sim 0.5 \mathrm{~K}$.

(3) The Yb moment of $\sim 0.1 \mu_{B}$ deduced from our measurements is 40 times smaller than the free-ion value, and is also much smaller than $\mathrm{Yb}$ crystal electric field (CEF) $\Gamma_{6}$ or $\Gamma_{7}$ doublet ground-state moments. ${ }^{12}$ Weak-moment static magnetism has also been observed in several heavyelectron systems with higher carrier densities. ${ }^{13}$

(4) The rapid $\mu^{+}$spin-lattice relaxation rates yield $\mathrm{Yb}$ spin correlation times which are anomalously long $\left[\tau_{c}\right.$ $-10^{3}-10^{4}\left(k_{B} T_{0} / \hbar\right)^{-1}$ ] in both kinds of domains.

The enormous low-temperature specific-heat $\gamma$ in $\mathrm{Yb}$ BiPt has been attributed to itinerant heavy-electron behavior. ${ }^{2}$ Our $\mu^{+}$SR observation of slow spin fluctuations and static magnetism in this compound raises the alternative possibility that a high density of low-lying magnetic excitations yields the large $\gamma$. This is the case for spin glasses, where a large temperature-linear term is found in the specific heat ${ }^{14}$ (although never as large as in the present case) and where $\mu^{+} \mathrm{SR}$ experiments [e.g., in AgMn (Ref. 15)] have confirmed the presence of a wide distribution of spin fluctuation rates. $\mu^{+} \mathrm{SR}$ relaxation behavior is qualitatively similar in $\mathrm{YbBiPt}$ and spin glasses.

*Permanent address: University of California, Riverside, CA 92521-0413.

'P. C. Canfield et al., J. Appl. Phys. 70, 5800 (1991).

${ }^{2}$ Z. Fisk et al., Phys. Rev. Lett. 67, 3310 (1991).

${ }^{3}$ A. Lacerda et al. (unpublished).

${ }^{4}$ For a description of the $\mu$ SR technique, see A. Schenck, Muon Spin Rotation Spectroscopy (Hilger, Bristol, 1985).

${ }^{5}$ See, for example, C. P. Slichter, Principles of Magnetic Resonance, 3rd ed. (Springer-Verlag, Berlin, 1990).

${ }^{6} \mathrm{R}$. Kubo and T. Toyabe, in Magnetic Resonance and Relaxation, edited by R. Blinc (North-Holland, Amsterdam, 1967), p. 810; see also R. S. Hayano et al., Phys. Rev. B 20, 850 (1979).

${ }^{7}$ P. C. Canfield and Z. Fisk, Philos. Mag. B (to be published).

${ }^{8}$ Spin-lattice relaxation yields an exponential relaxation function, as does the static distribution of local fields in a dilute
Our observation of differentiated types of magnetic domain may also shed light on the observed reduction ${ }^{3}$ of $\gamma$ in powdered $\mathrm{YbBiPt}$. We surmise that strain introduced by powdering creates the two types of domain (paramagnetic and frozen spin), one of which gives little or no contribution to the low-temperature $\gamma$. At high temperatures the paramagnetic domains dominate. The increase with decreasing temperature of the relative volume fraction of the frozen-spin domains might be due to a percolative process; the observed static spin freezing at $\sim 0.5 \mathrm{~K}$ could then signal the formation of an infinite cluster. Domains might be induced by strain dependence of the Kondo temperature, with paramagnetic and frozen-spin domains corresponding to regions where Kondo compensation or magnetic interactions, respectively, characterize the ground state.

Further $\mu^{+} \mathrm{SR}$ experiments using unstrained YbBiPt are currently in progress to address these issues. The association between the large $\gamma$ and static $\mathrm{Yb}$ magnetism would be strengthened by observation of such magnetism over the entire sample volume, particularly if the magnetism were disordered. If no static magnetism is observed in unstrained samples the large $\gamma$ must be due solely to heavy itinerant electrons. (The strain-induced domain picture for reduction of $\gamma$ would of course not hold if domain structure is also observed in unstrained high- $\gamma$ samples.) In either case the strain sensitivity of the magnetic and thermal properties underscores the very delicate balance between heavy-electron formation and magnetism in this system.

We acknowledge with thanks the expert help of C. Baines, D. Herlach, and the accelerator crew of PSI during the experiments, and have benefited from discussions with A. Lacerda, W. P. Beyermann, E. A. Knetsch, and J. A. Mydosh. Work at UC Riverside was supported in part by NSF Grants No. DMR-8814783 and No. DMR9114911, and the UC Riverside Academic Senate Committee on Research. Research at Leiden was supported financially by the Netherlands NWO and FOM. Work at Los Alamos was performed under the auspices of the U.S. Department of Energy. The financial support of the Schweizerische Nationalfonds is gratefully acknowledged.

spin glass [R. E. Walstedt and L. R. Walker, Phys. Rev. B 9, 4857 (1974)].

${ }^{9}$ Alternatively, the invisible component might reflect extremely rapid dynamics (short correlation times) leading to undetectably slow $\mu^{+}$relaxation. This seems unlikely, given the static component observed in zero field.

${ }^{10}$ S. Barth et al., Phys. Rev. B 39, 11695 (1989).

${ }^{\prime \prime}$ G. M. Luke et al., Bull. Am. Phys. Soc. 37 (1), 60 (1992).

${ }^{12}$ K. Sugawara, J. Phys. Soc. Jpn. 42, 1161 (1977).

${ }^{13}$ See, for example, D. E. MacLaughlin, in Encyclopedia of Physics, 2nd ed., edited by R. G. Lerner and G. L. Trigg ( $\mathrm{VCH}$, New York, 1991), p. 484, and references therein.

${ }^{14}$ L. E. Wenger and P. M. Keesom, Phys. Rev. B 11, 3497 (1975).

${ }^{15}$ D. E. MacLaughlin et al., Phys. Rev. Lett. 51, 927 (1983). 